\title{
Facet Joint Injections in Patients with Low Back Pains and Facet Arthropathies - Our Experience
}

\author{
Hussain Hadi Sharahili ${ }^{1}$, Khawaja Bilal Waheed ${ }^{2}$, Hawraa Ali Alaliwi ${ }^{3}$ \\ 1, 2, 3 Department of Radiology, King Fahad Military Medical Complex, Dhahran Saudi Arabia.
}

\section{ABSTRACT}

\section{BACKGROUND}

Chronic back pain is a common and frequent clinical presentation in any population. Presence of disc disease, facet syndrome, and vertebral body disease are usually addressed by the radiologists. Facet joints are proven to be the culprit in $15-45 \%$ of patients with low back pain. We wanted to compare effectiveness of fluoroscopy and computed tomography guided lumbar facet injections for pain relief in patients with facet arthropathies and mild canal stenoses.

\section{METHODS}

This is a retrospective cross-sectional study performed in the Department of Radiology at our Hospital in Dhahran. This record-based study was performed in our department from Jan. 2015 - 2020. All patients ( $\mathrm{N}=112)$ who underwent fluoroscopy and computed tomography (CT) guided facet injections (either alone or with epidural injections) for relief of chronic back pains (due to facet arthropathies and mild canal stenoses) were included, and grouped as (i) facet injections under fluoroscopy (F), and (ii) facet injections under CT guidance (C). Patients with acute disc prolapse, trauma to spine, lumbar surgeries, moderate to severe lumbar stenoses, spondylolisthesis, known systemic arthritides, those not suitable for the procedures, and those lost to follow-up were excluded. Repeat procedure within one year for recurrent complaints was used as a measure of effectiveness of the procedure. Proportional Z-test was used, and a p-value less than 0.05 was considered to be significant.

\section{RESULTS}

Out of a total of 112 patients, 64 were females (57\%) and 48 were males (43\%). The mean age was 56.4. Twenty out of 78 patients with facet injections under fluoroscopy and 3 out of 34 patients with facet injections under CT underwent repeat procedures $(\mathrm{P}=0.042)$.

\section{CONCLUSIONS}

CT guided facet injections combined with epidural injections may be more effective in relief of lower back pain in patients with facet arthropathies and mild canal stenoses.

\section{KEY WORDS}

Computed Tomography, Facet Block, Epidural Injection, Canal Stenosis
Corresponding Author: Dr. Khawaja Bilal Waheed. Consulant General Radiologist King Fahad Military Medical Complex (KFMMC) Dhahran - 31932, Saudi Arabia. E-mail: khawaja@kfmmc.med.sa DOI: 10.14260/jemds/2020/682

How to Cite This Article:

Sharahili HH, Waheed KB, Alaliwi HA. Facet joint injections in patients with low back pains and facet arthropathies - our experience. J Evolution Med Dent Sci 2020;9(42):3107-3111, DOI: 10.14260/jemds/2020/682

Submission 19-07-2020,

Peer Review 13-09-2020,

Acceptance 19-09-2020,

Published 19-10-2020.

Copyright (C) 2020 Hussain Hadi Sharahili et al. This is an open access article distributed under Creative Commons Attribution License [Attribution 4.0 International (CC BY 4.0)] 


\section{BACKGROUND}

Chronic back pain is a common and frequent clinical presentation in any population. ${ }^{1}$ It poses a diagnostic and therapeutic challenge due to multiple factors, overlapping clinical features and nonspecific radiological findings. The incidence of chronic back pain is at least $5 \%$ per annum, with an average prevalence of about $15 \%$ in adults. ${ }^{1}$ The association between symptoms and imaging findings is weak in such cases, therefore, many patients remain undiagnosed and continue to suffer pain. Structures that may cause low back pain include the vertebral body, inter-vertebral discs, the spinal cord, nerve roots, facet joints, ligaments, muscles, and sacroiliac joints. ${ }^{2}$ Presence of disc disease, facet syndrome, and vertebral body disease are usually addressed by the radiologists. Facet joints are proven to be the culprit in $15 \%$ $45 \%$ of patients with low back pain. ${ }^{3}$

Facet joint injection or facet block can be effectively performed by a radiologist as a pain relief method against facet arthropathy. ${ }^{4}$ Immediate pain relief after the injection identifies or confirms facet joints as the cause of spinal pain. Clinical suspicion of the facet syndrome, and focal tenderness over the facet joints with no significant disc disease are among major indications for facet injections. Except for relative limitations including local area skin conditions limiting injection (puncture site) or allergy to contrast (used for delineation of needle), no absolute contraindications have been documented. Local area skin conditions limiting injection (puncture site) or allergy to contrast (used for delineation of needle). No absolute contraindications have been documented. Similarly, lumbar facet joint injections may be repeated to reinstate pain relief without any deleterious effects. ${ }^{5}$ Image-guided injection of combination of local anaesthetic and steroid into or around the facet joint is meant to break the vicious cycle of inflammation, limited mobility and muscle spasm thereby providing pain relief. Therapeutic outcome is variable, though the procedure itself has shown to have high diagnostic accuracy, safety, and reproducibility.

Although immediate and short-term (3 - 6 months) beneficial effects of facet injections have been documented in literature.[4-6] However, very few studies have emphasized upon the long-term therapeutic results of facet injections, and their effectiveness under imaging guidance that can often be combined with epidural injections. ${ }^{6}$ Therefore, we aim to measure repetition rate (i.e., repeated facet joint lumbar intraarticular injection for recurrent complaints) as a measure of effectiveness of either fluoro - or CT - guided procedure for long - term (between 6 - 12 months) therapeutic pain relief at our Hospital.

\section{METHODS}

We performed a retrospective study involving secondary data analysis of patients' records in Radiology department at our Hospital in Dhahran between January 2015 to January 2020. All adult patients who had histories of chronic functionlimiting low back pains for at least 6 months duration and were diagnosed clinically and radiologically (by either computed tomography or magnetic resonance imaging) with facet arthropathies and mild (Grade - 1) lumbar canal stenoses (anteroposterior diameter of the canal less than $10 \mathrm{~mm}$, with separation of all cauda equina) ${ }^{7,8}$ were included, and evaluated for image guided (under fluoroscopy or CT) facet injections (with or without epidural injections) and repeat procedures within 6 - 12 month of initial injections.

Patients with acute disc prolapses, previous histories of trauma or injuries to spine, prior lumbar surgeries (discectomies, spino-laminectomies, or spinal fixations), moderate / severe (grade 2 / 3 lumbar stenosis; aggregated and not separated cauda equina) were excluded. Patients with spondylolisthesis (due to pars defects), with systemic inflammatory arthritides (like rheumatoid arthritis, or ankylosing spondylitis), not suitable for the procedures (e.g., those unable to lie in prone position), those with facet tropism (asymmetry between right and left facets, with one joint having more sagittal orientation than the other), and those lost to follow-up were also excluded.

Clinical information was obtained from patients' files / charts using Hospital Information System (HIS) and radiologic data about facet injections were retrieved from Radiology Information System / Picture Archiving and Communication System (RIS / PACS). Research protocol was approved by the Research Committee and need for informed written consents for the study was waived off considering retrospective nature of the study, already consented procedures, and nondisclosure of patients' information. All information was kept strictly confidential. Literature review was performed through electronic search (Google Scholar, PubMed).

All patients who underwent intra-articular facet injections (unilateral or bilateral; single or multiple segments) were grouped as: (i) Facet injections under fluoroscopy (F), and (ii) Facet injections under CT (C) guidance (either alone or combined with epidural injections). Facet injections usually involved 1.5- $2 \mathrm{ml}$ of combination of $40 \mathrm{mg}$ triamcinolone and 0.25-0.5 bupivacaine (for therapeutic purposes), while for epidural injections about $4-8 \mathrm{ml}$ of combination of $80 \mathrm{mg}$ triamcinolone and $0.25-0.5 \%$ bupivacaine hydrochloride (i.e., $2.5 \mathrm{mg} / \mathrm{mL}-5 \mathrm{mg} / \mathrm{mL}$ ) under fluoroscopy or CT guidance by a single operator (a dedicated musculoskeletal radiologist having more than 10 years of musculoskeletal imaging and intervention experience). All procedures were performed in sterile operating room (Fluoroscopy unit or CT suite) with intermittent scanning (for needle localization), while patients lying in prone positions with intravenous accesses, and light sedation with midazolam offered to all patients. Patients were followed up at 3, 6, and 12 months intervals at neurosurgery clinic (unless otherwise indicated), for need for a repeat procedure for recurrent or unsettling symptoms.

\section{Statistical Analysis}

Proportional analysis was performed using Z - test, and p value less than 0.05 was considered significant.

\section{RESULTS}

Out of total 112 patients, 64 were females ( $57 \%$ ) and 48 were males (43\%). Mean age of patients was 56.4 (range 32 - 84 years).

Adequate needle localizations were taken for successful facet [Figure - 1], and when combined with epidural [Figure - 
lumbosacral facet joint nerve block sessions or visits decreased at an annual rate of $0.2 \%$ from 2009 to 2018 and lumbosacral facet joint neurolysis sessions increased at an annual rate of $7.4 \%$ from 2009 to 2018 . Researchers have also found that lumbar medial branch radiofrequency ablations (RFAs) may provide benefit to well - selected individuals. ${ }^{12,13}$ with medial branch blocks (MBB) being more predictive than intra-articular (IA) injections. However, they cautioned more stringent selection criteria to improve denervation outcomes, but at the expense of more false negatives. ${ }^{10} \mathrm{Abd}$ - Elsayed $\mathrm{A}$ proposed that one prognostic block can be sufficient to move forward with radiofrequency ablation. ${ }^{12}$ Arıcı T and Kılıç E suggested that distal approach with an AP view (distal approach to place the needle parallel to the medial branch) for facet radiofrequency thermocoagulation is a viable alternative to other approaches. ${ }^{13}$

We observed a smaller number of patients for repeat procedures possibly due to adequate interventions. Onafowokan 00 et al reviewed strength of evidence available for multiple facet joint injections (FJIs) and medial branch blocks (MBBs) while reporting to NHS England. They found a paucity of levels I and II evidences available for the efficacy of multiple FJIs and MBBs in treating low back pain, with 'Getting it right first time' (GIRFT) data showing a high degree of variation in the use of multiple FJIs. ${ }^{5}$

Grading of facet arthropathy needs to be better assessed by computed tomography [Figure - 3], as MRI may underestimate the grading. ${ }^{2}$ Berg $\mathrm{L}$ et al while reviewing follow up imaging of 114 chronic back pain patients found that interobserver agreement on facet arthropathy (FA) severity based on facet joint space narrowing, osteophyte / hypertrophy, erosions, and subchondral cysts- was better with CT versus MRI. ${ }^{2}$ They observed that agreement was poorer for severity of osteophytes / hypertrophy than for the other evaluated FA findings with CT or MRI thus requiring more consistent grading of osteophytes / hypertrophy between different radiologists. Although we excluded patients with facet tropism. However, it should be noted that facet joint parameters may play an important role in the pathogenesis of recurrent lumbar disc herniation (rLDH). Li Z et al found that facet joint parameters (facet alignment and facet tropism) significantly influence the biomechanics of the corresponding segment.14 Song $\mathrm{Q}$ et al found that most facet joint $\mathrm{OA}$ appeared at the segment with intervertebral disc degeneration of more than grade III (sclerosis or moderate osteophytes). ${ }^{9}$ Facet joint OA was significantly exacerbated with the progression of disc degeneration grade. ${ }^{14}$ Facet tropism (divergence more than $7^{\circ}$ between the facet joint angles of both sides at the same segment) was also found significantly associated with lumbar disc degeneration, though we excluded such patients from our study.

Single center, small sample, single-operator, and retrospective analysis were few of important limitations of our study. Larger scale studies with more selective patients and pertinent lumbar facet parameters need to be addressed to evaluate more specific response and benefit of facet injections with graded approach from injection to ablation.

\section{CONCLUSIONS}

CT guided facet injections combined with epidural injections may be more effective in relief of lower back pain in patients with facet arthropathies and mild canal stenoses.

Data sharing statement provided by the authors is available with the full text of this article at jemds.com.

Financial or other competing interests: None.

Disclosure forms provided by the authors are available with the full text of this article at jemds.com.

\section{REFERENCES}

[1] Vining RD, Shannon ZK, Minkalis AL, et al. Current evidence for diagnosis of common conditions causing low back pain: systematic review and standardized terminology recommendations. J Manipulative Physiol Ther 2019;42(9):651-64.

[2] Berg L, Thoresen H, Neckelmann G, et al. Facet arthropathy evaluation: CT or MRI? Eur Radiol 2019;29(9):4990-8.

[3] Faber F. Lumbar facet joint disease: classification, clinical diagnostics, and minimally invasive treatment. Orthopade 2019;48(1):77-83.

[4] Manchikanti L, Sanapati MR, Pampati V, et al. Update of utilization patterns of facet joint interventions in managing spinal pain from 2000 to 2018 in the US fee-forservice medicare population. Pain Physician 2020;23(2):E133-49.

[5] Onafowokan 00, Fine NF, Brooks F, et al. Multiple injections for low back pain: what's the future? Eur Spine J 2020;29(3):564-78.

[6] Shim E, Lee JW, Lee E, et al. Facet joint injection versus epidural steroid injection for lumbar spinal stenosis: intra-individual study. Clin Radiol 2017;72(1):96.e7-14.

[7] Andreisek G, Imhof M, Wertli M, et al. A systematic review of semiquantitative and qualitative radiologic criteria for the diagnosis of lumbar spinal stenosis. AJR Am J Roentgenol 2013;201(5):W735-46.

[8] Mamisch N, Brumann M, Hodler J, et al. Radiologic criteria for the diagnosis of spinal stenosis: results of a Delphi survey. Radiology 2012;264(1):174-9.

[9] Song Q, Liu X, Chen DJ, et al. Evaluation of MRI and CT parameters to analyze the correlation between disc and facet joint degeneration in the lumbar three-joint complex. Medicine (Baltimore) 2019;98(40):e17336.

[10] Cohen SP, Bhaskar A, Bhatia A, et al. Consensus practice guidelines on interventions for lumbar facet joint pain from a multispecialty, international working group. Reg Anesth Pain Med 2020;45:424-67.

[11] Wu J, Zhou J, Liu C, et al. A prospective study comparing platelet-rich plasma and local anesthetic (LA)/corticosteroid in intra-articular injection for the treatment of lumbar facet joint syndrome. Pain Pract 2017;17(7):914-24.

[12] Abd-Elsayed A, Narel E, Loebertman M. Is a one prognostic block sufficient to proceed with radiofrequency ablation? A single center experience. Curr Pain Headache Rep 2020;24(6):23. 
[13] Arıcı T, Kılıç E. Distal approach for percutaneous radiofrequency thermocoagulation of lumbar medial branches in patients with lumbar facet arthropathy: a retrospective analysis. Agri 2020;32(1):31-7.
[14] Li Z, Gui G, Zhang Y, et al. Are facet joint parameters risk factors for recurrent lumbar disc herniation? A pilot study in a Chinese population. J Clin Neurosci 2020;77:36-40. 